\title{
Hemicraniectomy: A New Model for Human Electrophysiology with High Spatio-temporal Resolution
}

\author{
Bradley Voytek ${ }^{1}$, Lavi Secundo ${ }^{1}$, Aurelie Bidet-Caulet ${ }^{1}$, \\ Donatella Scabini ${ }^{1,2}$, Shirley I. Stiver ${ }^{2,3}$, Alisa D. Gean ${ }^{2,3}$, \\ Geoffrey T. Manley ${ }^{2,3}$, and Robert T. Knight ${ }^{1,2,3}$
}

\begin{abstract}
Human electrophysiological research is generally restricted to scalp EEG, magneto-encephalography, and intracranial electrophysiology. Here we examine a unique patient cohort that has undergone decompressive hemicraniectomy, a surgical procedure wherein a portion of the calvaria is removed for several months during which time the scalp overlies the brain without intervening bone. We quantify the differences in signals between electrodes over areas with no underlying skull and scalp EEG electrodes over the intact skull in the same subjects. Signals
\end{abstract}

\section{INTRODUCTION}

Hans Berger reported the results of the first human scalp EEG in 1925 from a 17-year-old boy with electrodes placed over a large surgical skull defect (Millett, 2001; Berger, 1929). These initial recordings were faint because of technical limitations, but for several years, EEG was only performed on patients with fissures or surgical holes in their skulls (Millett, 2001; Cobb, Guiloff, \& Cast, 1979). As Berger improved his EEG recording technology, he was able to acquire EEG from scalp electrodes over the intact skull and recordings from patients with skull defects diminished.

Despite its contributions to human cognitive neuroscience, scalp EEG has well-known limitations (Luck, 2005; Nunez \& Srinivasan, 2005). Scalp EEG has poor spatial localization and is susceptible to contamination from noise sources such as muscle activity that limit reliable acquisition of high-frequency neural activity. Further, the spectral amplitude of EEG signals is reduced as a function of frequency, resulting in substantial reductions in higher frequency power at scalp electrodes distant from the cortical surface.

Although early research on patients with skull defects was successful and lead to the discovery of the EEG, there has not been a systematic, within-subjects comparison of task-relevant EEG signals between electrodes placed over a large skull opening and those placed over the skull in

${ }^{1}$ University of California, Berkeley, CA, ${ }^{2}$ University of California, San Francisco, CA, ${ }^{3}$ San Francisco General Hospital over the hemicraniectomy have enhanced amplitude and greater task-related power at higher frequencies $(60-115 \mathrm{~Hz})$ compared with signals over skull. We also provide evidence of a metric for trial-by-trial EMG/EEG coupling that is effective over the hemicraniectomy but not intact skull at frequencies $>60 \mathrm{~Hz}$. Taken together, these results provide evidence that the hemicraniectomy model provides a means for studying neural dynamics in humans with enhanced spatial and temporal resolution.

human behavioral EEG. To address this issue, we examined three human participants who had undergone a surgical procedure wherein a portion of their skull was surgically removed for several months. This procedure, known as a decompressive hemicraniectomy, is an increasingly common, potentially life-saving neurosurgical procedure performed to reduce damage from an uncontrolled elevation in intracranial pressure (ICP) that frequently occurs following severe traumatic brain injury (TBI) and large-volume ischemic stroke. Many studies have shown that decompressive hemicraniectomy for elevated ICP in TBI and stroke reduces mortality and improves patient outcome (Yang et al., 2009; Huang et al., 2008; Juttler et al., 2007; Vahedi et al., 2007; Hofmeijer et al., 2006). In the subjects included in this analysis, a large portion of the calvaria was removed exposing the central sulcus and Sylvian fissure regions, providing a window devoid of bone over the primary motor and auditory cortices without contamination from underlying muscle activity. Thus, electrodes over the hemicraniectomy site are substantially closer to the underlying neural sources of the EEG. On the basis of the extant literature on breach rhythms, we hypothesized that that data from the electrodes placed over the hemicraniectomy site would show enhanced signal characteristics in comparison to electrodes placed over the intact skull.

In this report, we aimed to emphasize the improved quality of the electrophysiological signals recorded over the site of hemicraniectomy to highlight the utility of these patients for human cognitive neuroscience research. To this 
end, we compared electrodes over the hemicraniectomy with homologous electrodes over the intact skull. As with any new method, we first examined raw signal differences, with the hypothesis that, absent intervening skull and with electrodes closer to the cortical signal sources, raw time-series amplitudes would be larger, power across physiological frequency bands (theta, alpha, beta, and low and high gamma) would be greater, and noise in the higher frequencies often contaminated by muscle artifacts would be lower. Furthermore, without spatial smoothing due to influences of skull tissues and/or distance from source, we hypothesized that natural interelectrode correlations will be lower.

To test the utility of this method for human cognitive neuroscience we examined the effects of the hemicraniectomy on well-studied time-frequency and ERP electrophysiological measures with the hypothesis that task-related potentials will have greater amplitude and enhanced power across a wider frequency range. Because eye-movement artifacts are a common noise source in human behavioral electrophysiological studies, we also examined the effects of the hemicraniectomy on blink amplitudes with the hypothesis consistent with recent simultaneous scalp and intracranial EEG recordings (Ball, Kern, Mutschler, Aertsen, \& SchulzeBonhage, 2009) that blink amplitudes would be reduced over the hemicraniectomy.

Here we showed that signals recorded over the hemicraniectomy have higher spectral power, improved interelectrode spatial independence, reduced artifact susceptibility, and enhanced task-related power at higher frequencies (>60 Hz, high gamma; $\gamma_{\mathrm{H}}$ ) compared with homologous electrodes over intact skull. To demonstrate that the improved signal characteristics may serve a practical purpose, we also introduce a movement-related EEG interfrequency coupling metric that correlates with arm movement that is maximally effective using broadband $\gamma_{\mathrm{H}}$ over the site of the hemicraniectomy.

\section{METHODS}

\section{Subjects}

Because of the novelty of this method, we focused and reported on the various methodological challenges we faced. We initially recorded EEG from six participants (four males, two females), each of whom underwent surgical hemicraniectomy as a treatment for deteriorating neurological status resulting from increased ICP due to TBI. All craniectomies were performed at San Francisco General Hospital. At the time of study, each subject was an outpatient with at least 4 weeks recovery time following their decompressive surgery. All subjects gave informed consent in accordance to our study protocol approved by the University of California, Berkeley, and the UCSF Committees on Human Research. Subjects were tested in a sound-attenuated EEG recording room at the University of California, Berkeley in an outpatient setting.
Three subjects were eventually excluded from all analyses because of trauma-related complications mitigating the quality of EEG recording. One of these three subjects developed a diffuse extra-axial hygroma-a collection of proteinous cerebral spinal fluid on the outside of the brain-that can be present for several weeks to months postsurgery. This fluid increases the distance between the recording electrodes on the surface of the skin and the underlying brain, introducing noise and reducing signal strength. The two remaining subjects were excluded because of widespread cortical damage affecting both motor and auditory structures. All three of these subjects had reduced amplitude and noisy EEG signals making them unsuitable to test the main hypothesis. The three remaining subjects who form the basis for the current report were cognitively intact with minimal or no cortical damage at the sites of EEG recording, performed the two behavioral tasks well, and had reliable EEG signals. All three subjects examined in this report had intact scalp over the site of the hemicraniectomy at the time of EEG. None of these subjects had a significant hygroma at the time of testing, and none had any damage in motor or auditory cortices.

Subject 1 had a left hemicraniectomy; of the 64 scalp electrodes, 11 frontal to central-parietal electrodes were situated over the hemicraniectomy (electrode name and approximate underlying Brodmann's area (BA; Koessler et al., in press; Okamoto \& Dan, 2005): F3 (BA8), F5 (BA46), F7 (BA45), FC3 (BA6), FC5 (BA6), FT7 (BA22), C3 (BA1-2-3), C5 (BA1-2-3), T7 (BA21), CP5 (BA40), and TP7 (BA21). Subject 1 sustained a gunshot wound to the left frontal lobe anterior to premotor and motor cortices. His Glasgow coma scale (GCS) was 14 on admission. The patient was taken to the operating room for debridement of the gunshot wound, and a decompressive hemicraniectomy was performed to prevent further neurological deterioration from increasing ICP from the initial penetrating brain injury. At the time of EEG testing, his memory, attention, and motor function were normal and he did not have any frontal release signs. Of note, the patient is currently back to school.

Subject 2 had a right hemicraniectomy, and of the 64 scalp electrodes, 10 anterior frontal to central electrodes were over the hemicraniectomy: AF4 (BA9), AF8 (BA10), F4 (BA8), F6 (BA46), F8 (BA45), FC4 (BA6), FC6 (BA6), FT8 (BA22), C4 (BA1-2-3), and C6 (BA1-2-3). Subject 2 had accidentally fallen from three stories, and her GCS score was 3 in the field. She had a right subdural hematoma and right anterior temporal and orbito-frontal contusions. There was no evidence of injury to motor, auditory, or parietal cortices. Because of massive increase in ICP, she underwent an emergency decompressive hemicraniectomy. At the time of EEG testing, her memory, attention, and motor function were intact. She noted increased anxiety since the injury and was living with her parents.

Subject 3 had a left hemicraniectomy, and of the 64 scalp electrodes, 7 anterior frontal to central electrodes 
were over the hemicraniectomy: AF7 (BA10), F5 (BA46), F7 (BA45), FC5 (BA6), FT7 (BA22), C5 (BA1-2-3), and T7 (BA21). Subject 3 had fallen from two stories and sustained a left subdural hematoma. The patient had a GCS of 6 in the field that declined to a GCS of 3 on arrival to the ER. He underwent an emergency decompressive hemicraniectomy and removal of the subdural hematoma because of his rapid neurological deterioration. At the time of EEG testing, he was doing remarkably well and had no evidence of any residual neurological or behavioral deficits.

\section{EEG Recording}

EEG data were recorded using a $64+8$ channel BioSemi Active'Two amplifier (Metting van Rijn, Peper, \& Grimbergen, 1990). Horizontal eye movements (EOG) were recorded at both external canthi; blinks were monitored with a left inferior eye electrode and fronto-polar electrodes. EMG was recorded at the flexor digitorum sublimis muscle used during a particular movement block (left- or right-hand squeeze). All data-hemicraniectomy, skull, EMG, and EOG - were recorded on the same amplifier and were amplified ( $-3 \mathrm{~dB}$ at $\sim 819 \mathrm{~Hz}$ low-pass, DC coupled), digitized $(2048 \mathrm{~Hz})$, and stored for off-line analysis. Data were referenced off-line to the average potential of two earlobe electrodes and analyzed in MATLAB $®$ (R2008b, Natick, MA) using custom scripts and the EEGLAB toolbox (Delorme \& Makeig, 2004). Because of the location and the underlying brain exposure resulting from the hemicraniectomy, we chose two behavioral tasks during EEG recording known to activate primary motor and auditory cortices in an effort to assess the value of the hemicraniectomy model. The first task was a visually cued manual gripping task with simultaneously recorded EMG, and the second was a binaural auditory oddball task with tone deviant detection.
From a methodological standpoint, we encountered several issues directly related to recording EEG over the hemicraniectomy. Our recording system uses prefabricated, fitted caps (BioSemi, http://www.biosemi.com). After preparation with electroconductive gel, electrodes are snapped in place into the cap. Because this cap is elastic, when the cap is pulled over the head, the electrodes are pulled away from the hemicraniectomy because of the large concavity left by the missing piece of skull (see subject CT scans; Figure 1 and Video S1). On average, across the three subjects, the cortex-to-epidermis distance over the hemicraniectomy was reduced by $53.4 \%$ as compared with over the skull $(p<.001$; calculated for each subject as extracted from multiple measurements over absent and intact skull).

To obtain good electrode contact, the method that gave us the best signal stability involved placing a gauze mesh around the head over the electrode cap; this mesh pulled the hemicraniectomy electrodes against the surface of the skin. Snapping electrodes into the cap also proved difficult over the hemicraniectomy site. Without the normal force from the skull pushing back during electrode placement, we had to be particularly careful, leading to substantially increased setup times. Use of a hemostat to hold the cap electrode attachments was a valuable method to safely speedup electrode attachment. Once the electrodes were snapped into place, cotton balls were placed over the hemicraniectomy electrodes, under the gauze mesh, to hold them in place.

\section{Auditory Task}

Subjects listened to a sequence of standard $(p=.79)$ and target $(p=.21$ ) sounds and were instructed to press the space bar on a keyboard after hearing a target. Standard
Figure 1. CT scans of the three subjects demonstrating the extent of the hemicraniectomy. The upper row images were reconstructed using "Surface 3-D volume-rendered" multidetector helical CT data. The lower row images include $\mathrm{AP}$ and lateral plain radiographs and an axial CT image. Note how the calvarial defect (arrows) includes virtually the entire the frontal bone with relative sparing of the supraorbital rim and paramedian skull. It extends into the parietal bone and squamosal portion of the temporal bone down to just above the ear. The axial CT image demonstrates the characteristic concave deformity seen in many patients before cranioplasty. Note the immediate proximity of the skin surface to the underlying normal brain

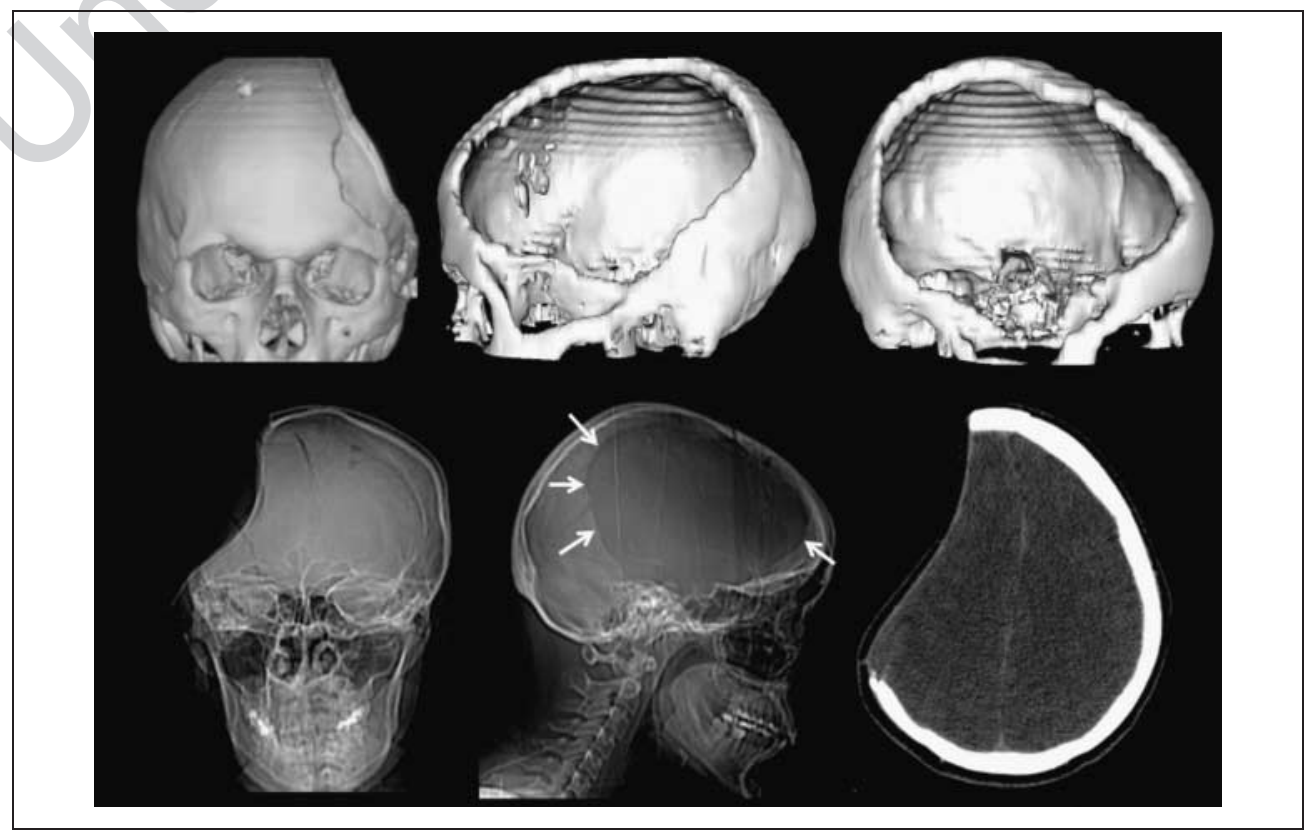


sounds were 50-msec band-passed noise bursts (554$740 \mathrm{~Hz}$ ), whereas target sounds were 50-msec pure tones of $988 \mathrm{~Hz}$. The intertrial interval (ITI) between successive sounds was jittered between 700 and $900 \mathrm{msec}$. All sounds were presented through two speakers positioned in front of the subject on their left and right sides at an intensity level judged comfortable by the patient at the beginning of the experiment (approximately $70 \mathrm{~dB}$ ).

\section{Motor Task}

Subjects were seated in front of a computer monitor while holding a spring grip device in either their left or right hand depending on block. Subjects fixated on a white central cross and were instructed to squeeze the gripper when they saw the go cue (whenever the cross flashed green). The ITI was jittered between 1500 and 2000 msec. EMG was recorded as the difference between two electrodes placed approximately $1 \mathrm{~cm}$ apart along the flexor digitorum sublimis muscle of the gripping hand.

\section{Spontaneous EEG}

All root mean square (RMS) calculations were performed on band-pass filtered raw EEG data from all three subjects across all conditions across all hemicraniectomy electrodes and their homologous skull electrodes. For the "raw data" analysis, a 0.1- to 30-Hz passband was used. For each subject, 500 random, 1-sec data segments were pulled from a random pair of homologous band-passed hemicraniectomy and skull electrodes, and artifact trials were removed as normal; RMS values were then calculated for each segment. A similar method was used for the interelectrode correlation analysis using a passband of 0.1$30 \mathrm{~Hz}$ and 500 random 1-sec data segments per subject. Pearson correlation coefficients were calculated on an all-to-all basis for hemicraniectomy and homologous skull electrodes and binned as a function of interelectrode distance. EMG-contaminated electrodes were classified using three methods outlined in Fu, Daly, and Çavuşoğlu (2006) and Goncharova, McFarland, Vaughn, and Wolpaw (2003): (1) electrodes AF7/8 and FT7/8, which generally sit over frontalis or temporalis muscles; (2) visual inspection for regular "railroad cross-tie" EMG spiking activity; and (3) gamma bursting during facial and jaw muscle tensing or biting.

\section{Blinks}

To calculate the effects of hemicraniectomy on blink amplitude propagation - a common noise source in scalp and intracranial EEG (see Ball et al., 2009)—we time locked to all naturally occurring blinks across all three subjects by thresholding the product of the inferior and superior eye electrodes, giving a total of 1344 blink trials for the three subjects. To identify blinks, we made use of a semiautomatic detection algorithm. First, the inferior and superior EOG channels were point-by-point multiplied, creating a surrogate blink channel with amplified eye blinks. We also created a surrogate saccade channel by point-by-point multiplication of the left and right horizontal eye channels. Saccades generally have smaller amplitudes and different time courses than blinks, but both blinks and saccades are easy to visually identify in their surrogate channels. For each subject, we found several canonical blinks in the surrogate blink channel judged by visual inspection of waveforms and scalp topographies, which look different for blinks than for saccades. We then used the amplitude of the surrogate blink time series at those canonical trials to set the threshold for the detection algorithm that sweeps through the surrogate channel to identify time points of putative blinks. Once putative blinks are found, we created epochs around those time points and removed nonblink artifacts thorough visual inspection of both the vertical and the horizontal surrogate eye channels. For each trial, the data were normalized to the maximum value across all electrodes during that trial.

\section{Time-frequency Analyses}

For event-related spectral perturbation (ERSP) plots, data were band-pass filtered across 75 passbands in 2-Hz increments from 0 to $150 \mathrm{~Hz}$. We used a Gaussian-shaped filter by performing point-by-point multiplication of a Gaussian with the FFT of the signal. The Gaussian standard deviation was $10 \%$ of the center frequency resulting in FWHM of 0.2355 of the center frequency. The analytic amplitude (absolute value of the Hilbert transform) for each passband was used to create a grand average time-frequency ERP (ERP ${ }_{\text {tf }}$; see Bruns (2004) for comparison of timefrequency decomposition methodologies). After obtaining the real $\mathrm{ERP}_{\mathrm{tf}}$ for each frequency band, stimulus onset times were randomly shuffled-keeping the real ITIs fixed - and a surrogate passband $\mathrm{ERP}_{\mathrm{tf}}$ was created using the same window length. One thousand surrogate grand average ERPs ${ }_{t f}$ were created for each passband, giving a probability distribution of amplitude values for each passband from the data itself. From these surrogate ERPs, a $z$ score was calculated at each time point for each passband creating an ERSP across the frequencies of interest.

\section{Auditory Analysis}

For ERP analyses, EEG was filtered between 0.1 and $30 \mathrm{~Hz}$, and the data were segmented from $100 \mathrm{msec}$ before sound onset to 1000 msec after using a 100-msec prestimulus baseline. Trials contaminated by blinks or eye movements were removed from analysis. For each subject, for each trial, the extremum value (maximum for P50 and P200, minimum for N100) within the component time window was found; these values were then subjected to statistical analysis. For Video S2, the grand average for each time point across the three subjects was used. For scalp topographies in Figures 3A and 4B, the mean amplitude across each component time range was used. ERP component time ranges 
Figure 2. Blink artifact propagation. (A) Scalp topographies of mean peri-blink amplitude for all three hemicraniectomy subjects compared with three young controls with intact skull, demonstrating the blink amplitude drop over the hemicraniectomy electrodes. Electrodes over the site of hemicraniectomy in any one subject are in white. (B) Peri-blink amplitudes from the eye. $* * * p<.001$, $* p=.042$, statistically significant differences; bars are SEM. as a function of distance

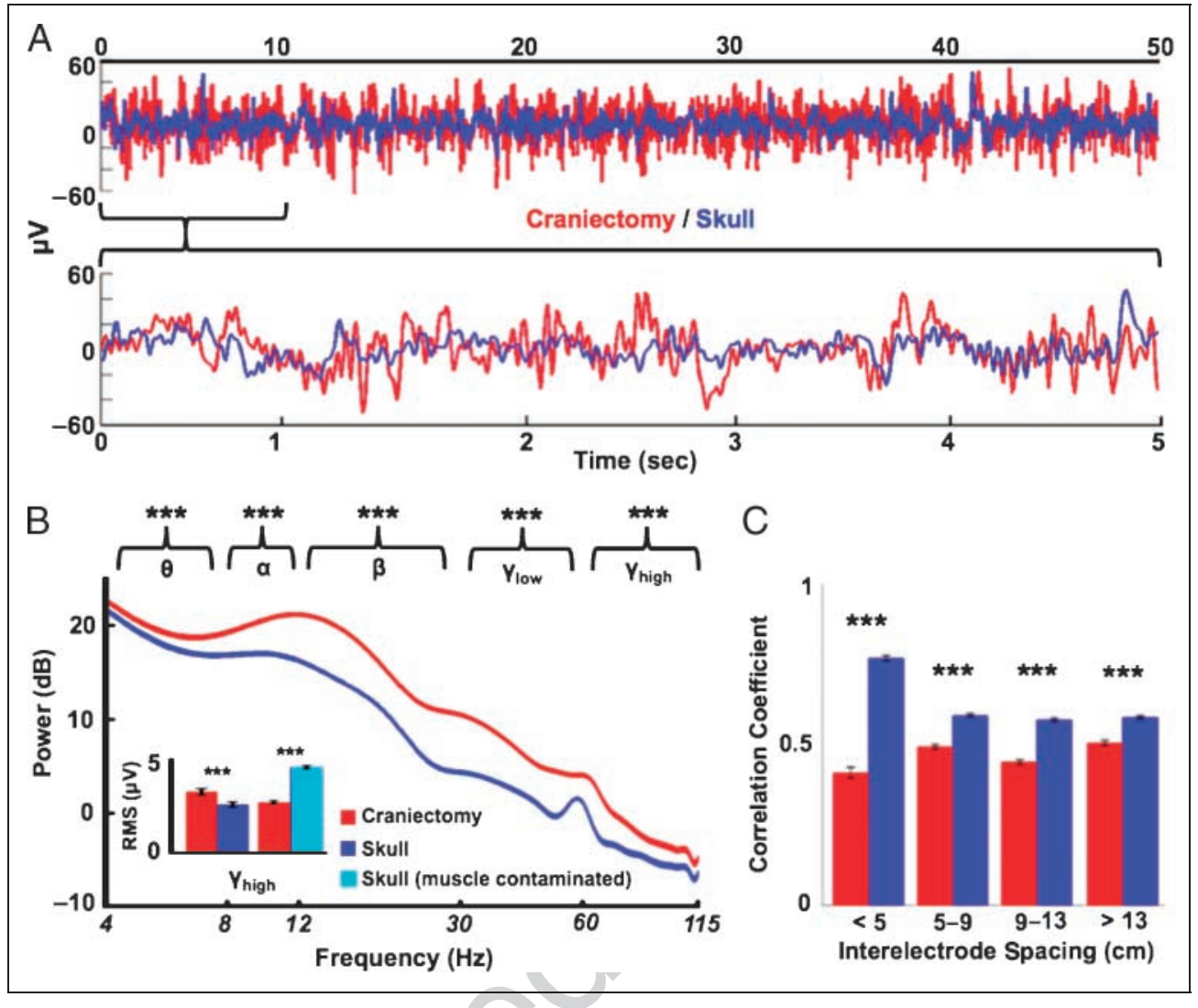

were 30-80 msec for P50, 70-160 msec for N100, and 150260 msec for P200. For $\gamma_{\mathrm{H}^{-}}$and $\beta$-band analyses, the analytic amplitude of band-passed signals were calculated $(\beta, 12$ $30 \mathrm{~Hz} ; \gamma_{\mathrm{H}}, 65-115 \mathrm{~Hz}$ ), and data were then analyzed similar to ERP analysis. For $\beta$-band analyses, the minimum value between 300 and 500 msec poststimulus onset was used to index activity for each trial; for $\gamma_{\mathrm{H}}$-band analyses, the maximum between 100 and 300 msec was used.

Electrodes for each ERP component were selected on the basis of whichever hemicraniectomy/skull pair gave the largest mean response for the component of interest across each component's a priori time range. For time-frequency analyses, electrode pairs that gave the largest $\gamma_{\mathrm{H}}$ response were compared (though it is important to note that no skull electrodes showed significant $\gamma_{\mathrm{H}}$ response to targets or tones). The electrode pairs used for each component for each subject as well as the corresponding number of trials $(n)$ after artifact rejection are as follows:

- Subject 1 ( $n=143)$ : P50 (C5/6), N100 (C5/6), P200 $(\mathrm{T} 7 / 8), \gamma_{\mathrm{H}} / \mathrm{\beta}(\mathrm{CP} 3 / 4)$;

- Subject 2 ( $n=141)$ : P50 (FC3/4), N100 (FC5/6), P200 (FC3/4); and

- Subject 3 ( $n=144)$ : P50 (C3/4), N100 (FC5/6), P200 $(\mathrm{FC} 3 / 4), \gamma_{\mathrm{H}} / \beta(\mathrm{FC} 5 / 6)$.

Because of the large amount of high-frequency noise in the homologous skull electrode for Subject 2, auditory time-frequency analyses were performed on Subjects 1 and 3 only.

\section{Motor Analysis}

Movement onset and offset were indexed from EMG data conditioned using the Teager-Kaiser energy operator (see Solnik, DeVita, Rider, Long, \& Hortobágyi, 2008), where $x_{t}=x_{t}^{2}-$ $\left(x_{t}-1\right)\left(x_{t}+1\right)$. The first derivative (element-by-element difference) of a logical vector of EMG values surpassing a threshold was calculated giving instantaneous movement onset and offset times. For motor $\beta$ and $\gamma_{H}$ ERP analyses, we selected electrode pairs in the same manner as in the auditory ERP analyses, choosing the electrode pairs that gave us the largest $\beta$-band amplitude decrease relative to baseline during contralateral hand movement. Hemispheric differences were calculated by finding the minimum (for $\beta$-band) or maximum (for $\gamma_{\mathrm{H}}$-band) values during the movement. For $\beta$-band analyses, the minimum value between 100 and $500 \mathrm{msec}$ postmovement onset was used to index activity for each trial; for $\gamma_{\mathrm{H}}$-band analyses, the maximum between 0 and $300 \mathrm{msec}$ was used. The electrode pairs used as well as the $n$ for each contra- or ipsi-craniectomy hand movement are as follows:

- Subject 1 (C3/4): $n=78$ contra-craniectomy, $n=85$ ipsi-craniectomy hand;

- Subject 2 (C3/4): $n=64$ contra-craniectomy, $n=66$ ipsi-craniectomy hand; and

- Subject 3 (C5/6): $n=102$ contra-craniectomy, $n=113$ ipsi-craniectomy hand.

ERSPs were calculated using the same procedure as auditory ERSP analysis, using the same electrode pairs used for $\mathrm{ERP}_{\mathrm{tf}}$ analyses with a window from $1300 \mathrm{msec}$ 


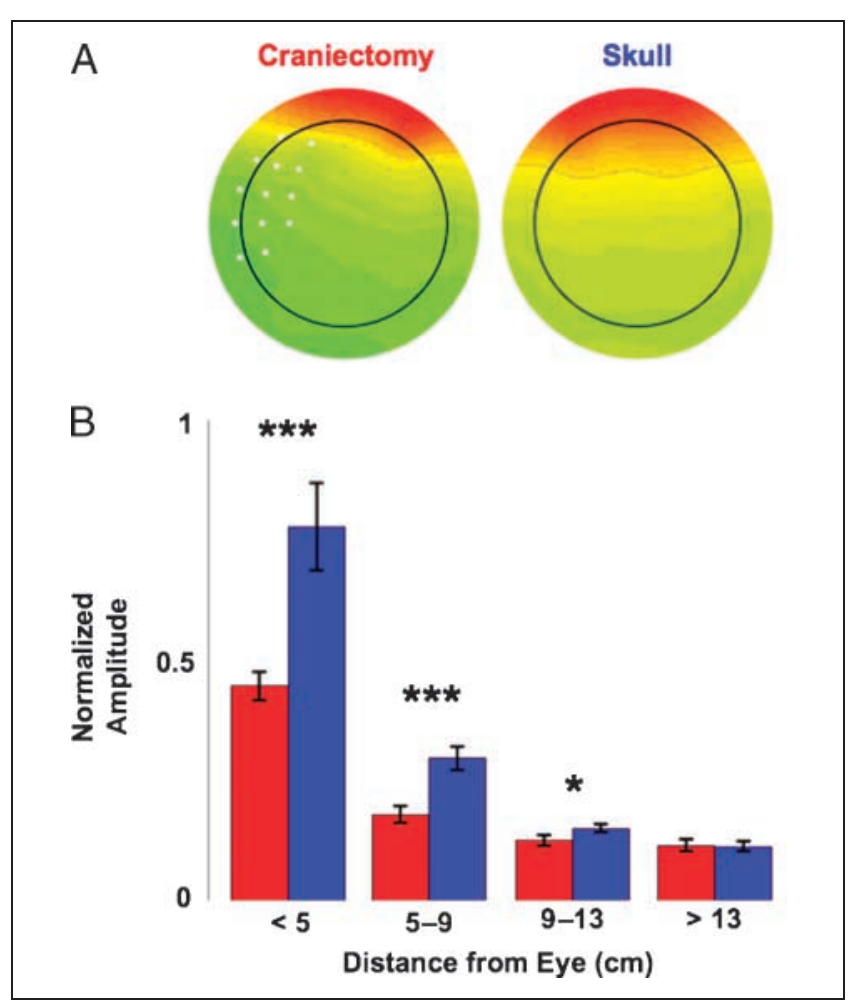

Figure 3. Auditory ERPs in response to correctly identified infrequent deviant tones. (A) Peak ERP amplitudes for components P50, N100, and P200 over hemicraniectomy and homologous skull sites for each subject. (B) Grand average scalp topographies of mean amplitude across the time range of each ERP for all three subjects. $* * * p<.001$, ${ }^{*} p=.033$, statistically significant differences; bars are SEM.

before to 500 msec after movement onset and a baseline from 1300 to 1000 msec before movement onset.

\section{Interfrequency Coupling}

Interfrequency coupling indices were calculated using the 10-Hz low-pass filtered $\gamma_{\mathrm{H}}$ and $\beta$ analytic amplitudes of band-passed data for all three subjects using the same electrodes used in the motor task. Band-pass filtered (20-120 Hz) EMG data were smoothed using the TeagerKaiser energy operator algorithm as above. To calculate the gamma-band index (GBI), the entire $\beta$ amplitude vector was shifted forward by the median RT for each subject, in each condition, and then divided, element by element, by the $\gamma$ amplitude vector of the same electrode to compensate for the relative differences in onset times between bands. Without this shift, GBI/EMG coupling still performs better over hemicraniectomy electrodes than skull $(p<$ .001, all comparisons) and better than $\gamma_{\mathrm{L}} / \beta$ and $\gamma_{\mathrm{H}}$ alone ( $p<.001$, both comparisons) but more similar to $\beta$ alone $(p=.18)$. Trial-by-trial Pearson correlation coefficients were calculated between the movement index at the electrode contralateral to the movement hand and the smoothed EMG from that hand, using a window from 500 msec before to $500 \mathrm{msec}$ after movement onset for all subjects.

\section{Statistical Analyses}

Significance for all hemicraniectomy versus skull electrode comparisons in the RMS, interelectrode correlation, ERP, $\mathrm{ERP}_{\mathrm{tf}}, \mathrm{EEG} / \mathrm{EMG}$ correlation, and blink comparison analyses was achieved using a two-sided Wilcoxon rank sum test. For all scalp topographies (Figures 3A and 4B and Video S2), electrodes in Subject 2 were digitally swapped across the midline to normalize all electrodes over hemicraniectomy sites to the left hemisphere.

\section{RESULTS}

\section{Spontaneous EEG}

RMS activity of the raw time-series data was larger at electrodes over the site of hemicraniectomy as compared with homologous skull electrodes $(p<.001)$. Similarly, RMS activity for all examined frequency bands was larger over the hemicraniectomy compared with the skull $(p<.001$ all comparisons; see power spectra in Figure $2 \mathrm{~B}$ ). It is important to note that $\gamma_{\mathrm{H}}(65-115 \mathrm{~Hz})$ hemicraniectomy RMS activity was greater only when compared with skull electrodes not contaminated by underlying muscle activity, whereas muscle-contaminated skull electrodes show larger RMS compared with their homologous hemicraniectomy electrodes $(p<.001$; Figure 2B).

To quantify the extent to which the skull causes spatial smoothing, we examined natural (task-irrelevant) interelectrode time-series correlations in an all-to-all manner for hemicraniectomy electrodes and homologous skull electrodes. Interelectrode correlations were binned into four groups according to interelectrode distance. For all distances, interelectrode correlations were lower over hemicraniectomy sites compared with skull $(p<.001$ all comparisons; Figure 2C).

Blink artifacts are a common noise source in scalp EEG recordings. Eye movements produce large-amplitude fluctuations that propagate long distances, detectable to some degree even at posterior scalp electrodes. We tested the artifact propagation properties of the skull by time locking the EEG signal to all naturally occurring blinks in all three subjects. Extracted blink amplitudes are largest at electrodes closest to the eyes and drop off rapidly as a function of distance. Similar to the interelectrode correlation analysis, blink amplitudes were binned according to distance from the eye. For all electrodes within $13 \mathrm{~cm}$ of the eye, blink amplitudes were smaller over the hemicraniectomy hemisphere compared with homologous sites over intact skull $(p<.001$ at $<9 \mathrm{~cm}, p=.042$ between 9 and $13 \mathrm{~cm}$; Figure 3$)$.

\section{Auditory Responses}

Because different ERP components had restricted spatial distributions over the hemicraniectomy side (Figure 4B), analyses were conducted on a single-electrode basis. ERPs were compared over the pair of homologous electrodes that yielded the largest mean normalized activity across 
Figure 4. Comparison of raw signals. (A) Time-series data has more power and decreased signal redundancy over the hemicraniectomy (red) as compared with the skull (blue). The top trace shows $50 \mathrm{sec}$ of raw data from two homologous electrodes from Subject 1 (F3/ $\mathrm{F} 4)$; the bottom is zoomed in on the first $5 \mathrm{sec}$ of the top trace. (B) Mean power spectra for hemicraniectomy and skull electrodes not contaminated by muscle noise (width of spectrum indicates SEM for each frequency; not $60 \mathrm{~Hz}$ contamination). Asterisks above each band grouping indicate RMS amplitude for that frequency band is greater for hemicraniectomy electrodes compared with homologous skull electrodes. For $\gamma_{\mathrm{H}}(65-115 \mathrm{~Hz})$ RMS analyses, electrodes were divided into two groups: skull electrodes with high muscle noise and those with low (inset; see Supplemental methods for muscle noise classification methods). $\gamma_{\mathrm{H}}$ is greater over hemicraniectomy sites compared with skull electrodes only when skull electrodes are not contaminated by muscle noise. (C) Interelectrode correlations for all hemicraniectomy pairs are lower at all distances compared with homologous skull pairs. $* * * p<.001$, statistically significant differences; bars are SEM.

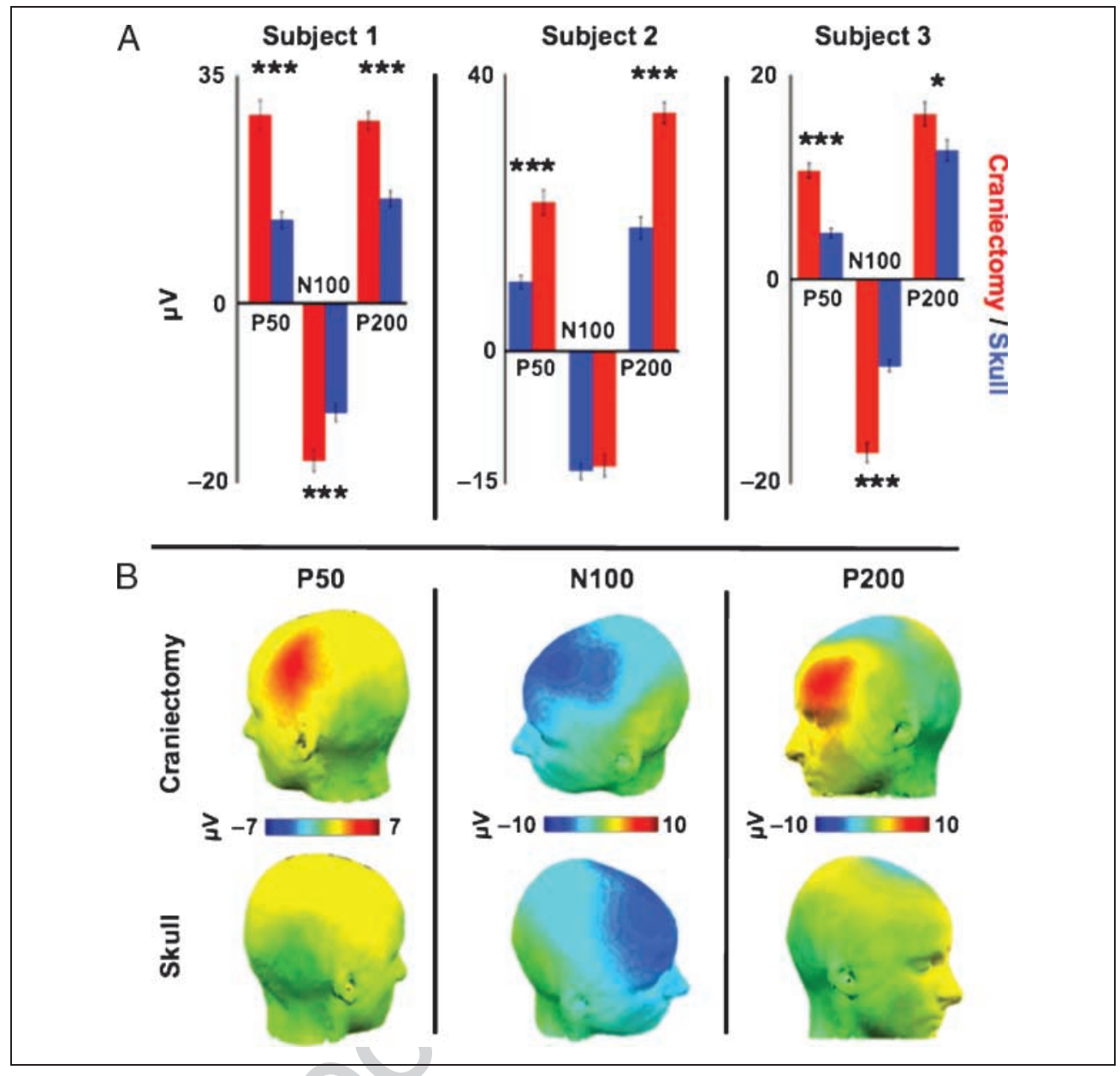

the a priori time range of interest for each component. For the oddball task, we examined three auditory ERPs evoked by infrequent deviant tones: P50, N100, and P200 (Figure 4A and Video S2). Video S2 shows the time course of EEG responses to deviant tones. Approximately 12-15 msec after tone onset, a focused positivity can be seen lateralized to electrodes over the hemicraniectomy, followed by a frontal negativity and positivity biased toward the craniectomy hemisphere. P50 magnitude was larger in all three participants over the site of the hemicraniectomy ( $p<.001$ all subjects), N100 amplitude was larger over hemicraniectomy in Subjects 1 and $3(p<.001$ both subjects), and P200 magnitude was larger in all three subjects $(p<.001$ Subjects 1 and $2, p=.033$ Subject 3$)$. In this task, subjects were fast at detecting the targets (RTs around 250-300 msec). Thus, long-latency target-related ERPs such as the P300 were superimposed by ERPs related to the motor response and were not reliably observed.

Auditory stimulus-locked ERSPs were calculated for 75 equally spaced frequency bands between 0 and $150 \mathrm{~Hz}$ for Subjects 1 and 3. Subject 2 was excluded from auditory time-frequency analyses because of strong, high-frequency muscle contamination over intact skull electrodes. Targetrelated $\gamma_{\mathrm{H}}$ was higher over hemicraniectomy compared with skull ( $p=.0032$; Figure $5 \mathrm{~A}$ and $\mathrm{B})$. There was also a robust lower frequency, $\beta$-band $(12-30 \mathrm{~Hz}$ ) amplitude decrease to targets compared with standard stimuli over the hemicraniectomy $(p<.001)$. The magnitude of this decrease was larger over the hemicraniectomy compared with homologous skull electrodes $(p<.001$; Figure $5 \mathrm{C}$ and D).

\section{Motor Time-frequency Responses}

Movement-locked ERSPs were calculated for all three subjects (Figure 6A and B). We focused on two specific frequency bands $-\beta$ and $\gamma_{\mathrm{H}}$ - in accordance with previous findings (Darvas et al., in press; Dalal et al., 2008; Neuper, Wörtz, \& Pfurtscheller, 2006; Crone, Miglioretti, Gordon, \& Lesser, 1998; Crone, Miglioretti, Gordon, Sieracki, et al., 1998). Single-trial analysis of the activity in these bands shows a robust, trial-by-trial event-related profile over 
Figure 5. Stimulus-locked auditory time-frequency activity. (A and B) ERSPs for Subjects 1 and 3 over homologous hemicraniectomy and skull sites showing target-related $\gamma_{\mathrm{H}}$ over the hemicraniectomy. ( $\mathrm{C}$ and D) Same as panels A and B to nontarget auditory stimuli. Note that the early cluster of $\gamma_{\mathrm{H}}$ starting at approximately 100 msec poststimulus onset is only present over the hemicraniectomy in response to targets.

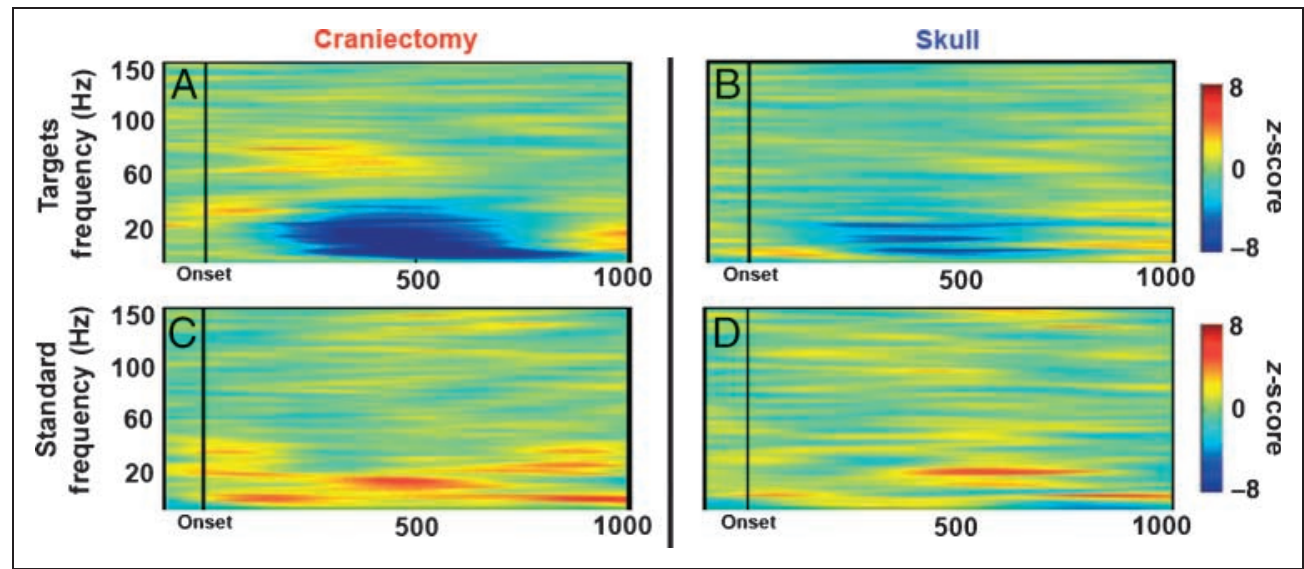

the hemicraniectomy (see Figure 6C-F). Across all three subjects, movement-related $\beta$ and $\gamma_{\mathrm{H}}$ magnitudes were larger over hemicraniectomy compared with skull $(p<$ .001 both comparisons; Figure $6 \mathrm{G}$ and $\mathrm{H}$ ).

\section{Interfrequency Coupling in Movement}

Research on movement-related scalp EEG, magnetoencephalography (MEG), and intracranial electrocortico- graphy (ECoG) oscillations has shown robust $\beta$ power decrease (desynchronization) beginning before the onset of and extending through the duration of movement (Pfurtscheller \& Lopes da Silva, 1999). Several studies have also indicated a relationship between movement-related $\beta$-decrease and $\gamma$-increase during real and imagined movements (de Lange, Jensen, Bauer, \& Toni, 2008; Pfurtscheller $\&$ Lopes da Silva, 1999). Given the known roles of $\beta$ decrease and $\gamma$-increase with movement and the relative
Figure 6. Contralateral movement-related time-frequency activity. (Note that panels $\mathrm{A}-\mathrm{F}$ are on the same $x$-axis scale). (A and B) ERSPs for all three subjects over homologous hemicraniectomy and skull sites showing movement-related $\gamma_{\mathrm{H}}$ over the hemicraniectomy. (C and D) Single trial $\beta(12-30 \mathrm{~Hz})$ and (E and F) $\gamma_{\mathrm{H}}(65-115 \mathrm{~Hz})$ movement-related activity in Subject 1 locked to movement onset-sorted by movement offset-illustrating the trial-by-trial power at hemicraniectomy electrode (C3) compared with the homologous skull electrode (C4). (G and $\mathrm{H}$ ) $\beta$ and $\gamma_{\mathrm{H}}$ ERPs for all subjects.

Movement-related $\beta$ and $\gamma_{H}$ are greater over hemicraniectomy electrodes as compared with homologous skull sites. $* * * p<.001$, statistically significant differences.

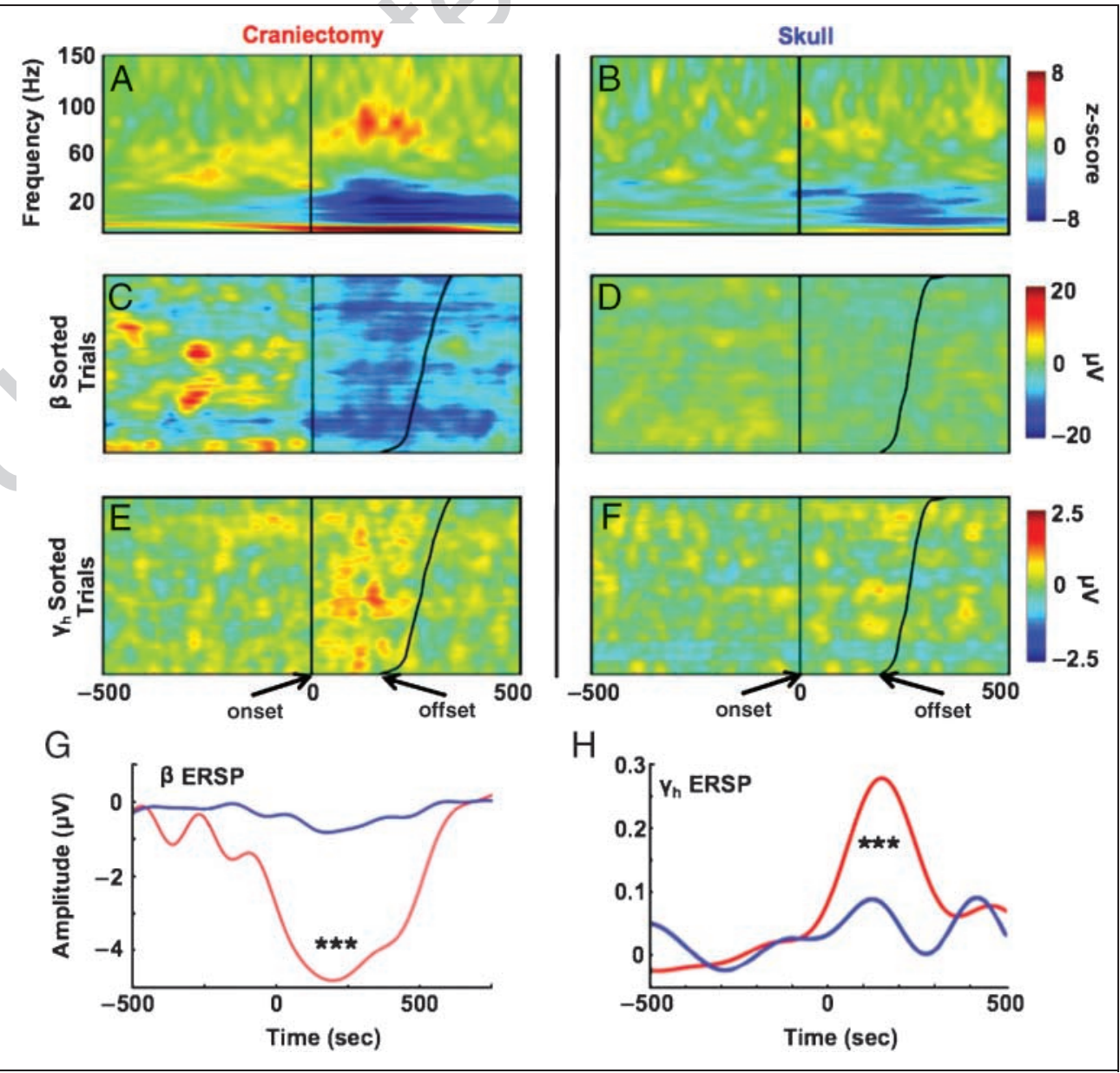


robustness of trial-by-trial $\beta$ and $\gamma_{\mathrm{H}}$ movement-related activity over the site of hemicraniectomy, we assessed the relationship between these two bands with movement via a GBI that tracked motor behavior in the hemicraniectomy patients.

We examined the relationship between muscle activity (as indexed by EMG) and $\gamma_{\mathrm{H}} / \beta$ interfrequency coupling to assess whether this metric provided a better measure of movement than either band alone. We defined the GBI as the division of $\gamma_{\mathrm{H}}$ by $\beta$ activity within an electrode: If $\gamma_{\mathrm{H}}$ increases while $\beta$ decreases, GBI is larger. We found a robust trial-by-trial correlation between GBI recorded over the hemicraniectomy with the EMG recorded over the active contralateral muscle groups (see example traces in Figure 7A). Trial-by-trial GBI/EMG correlations are larger over the site of hemicraniectomy $(p<.001)$, with the hemicraniectomy GBI performing better than $\gamma_{\mathrm{H}} /$ EMG or $\beta /$ EMG correlations ( $p<.001$ both comparisons; Figure $7 \mathrm{~B}$ and $\mathrm{C}$ ). To assess whether this index is dependent on $\gamma_{\mathrm{H}}$ specifically and not just $\gamma$ in general, we calculated the same correlation using a low-gamma $\left(\gamma_{\mathrm{L}}\right)$ band (30-55 Hz) separately. This hemicraniectomy $\gamma_{\mathrm{L}} / \beta$ index correlated less with EMG than the high-gamma metric $(p<.001$; Figure 7B).

\section{DISCUSSION}

EEG in subjects with skull defects has been previously reported as the "breach rhythm" (Cobb et al., 1979; Cobb \& Sears, 1960). These studies showed that breach rhythm signals were higher in overall power compared with normal scalp EEG in agreement with our findings. These earlier studies examined the effects of the skull on scalp electrical recordings, showing that the skull acts as a spatial filter smoothing underlying signals (Cobb \& Sears, 1960) and averaging electrical potentials from an extended patch of cortex (DeLucchi, Garoutee, \& Aird, 1962). Because of the improved signal quality and spatial localization, many researchers are now recording EEG signals from electrodes implanted directly on the cortical surface of patients undergoing brain surgery. These ECoG signals have improved power, increased bandwidth extending into the $\gamma_{\mathrm{H}}$ range, and improved spatial localization compared with scalp EEG. Practically, researchers are making use of the improved signal from invasive recordings to drive brainmachine interface (BMI) devices to assist people with paralysis (Miller et al., 2007; Hochberg et al., 2006). Recent intracranial and scalp EEG and MEG research indicates that task-relevant $\gamma_{\mathrm{H}}$ oscillations are generated in each of
Figure 7. $\mathrm{EEG} / \mathrm{EMG}$

movement correlation.

(A) Example of raw traces of EMG and contralateral $\gamma_{\mathrm{H}} / \mathrm{B}$ index in Subject 1 over hemicraniectomy electrode C3. (B) Average trial-by-trial EMG/ EEG correlations for all subjects. For all correlation bands, hemicraniectomy electrodes perform better than skull electrodes. For hemicraniectomy electrodes, $\gamma_{\mathrm{H}} / \beta$ correlates higher with movement than $\gamma_{\mathrm{L}} / \beta, \beta$-only, and $\gamma_{\mathrm{H}^{-} \text {only. (C) }}$ Single-trial movement-locked EMG and contralateral $\gamma_{\mathrm{H}} / \beta$ index in Subject 1 over hemicraniectomy (C3) and skull (C4), illustrating trial-by-trial correlation between $\gamma_{\mathrm{H}} / \beta$ index and EMG. $* * * p<.001$, statistically significant differences; bars are SEM.

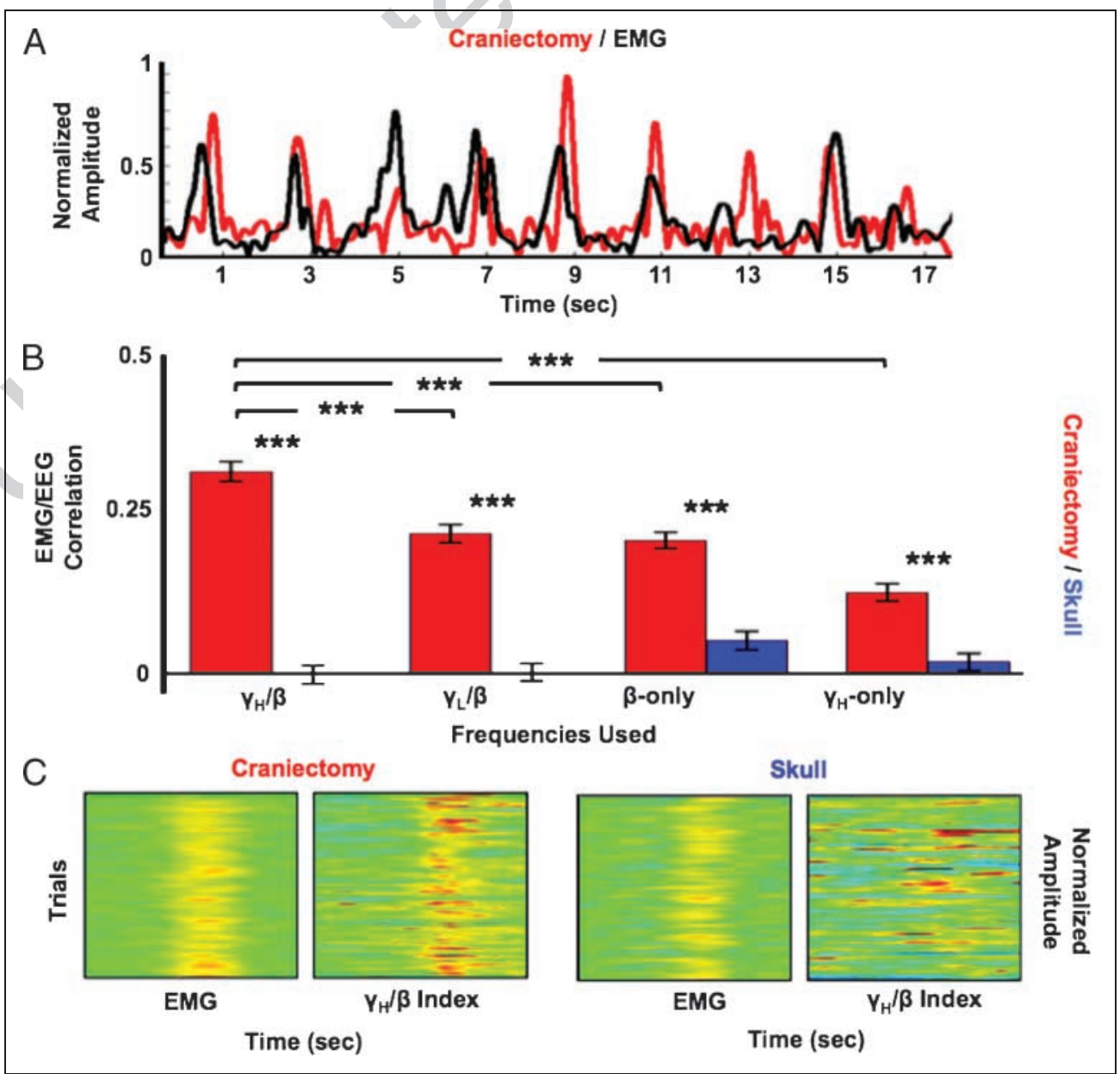


these tasks (Ball et al., 2008; Dalal et al., 2008; Edwards, Soltani, Deouell, Berger, \& Knight, 2005; Crone, Miglioretti, Gordon, Sieracki, et al., 1998). It is important to emphasize that these high-frequency oscillations are emerging as important markers for a variety of cognitive and behavioral functions.

Power in human EEG drops off as a function of distance and is inversely proportional to frequency in a $1 / f$-like relationship (Bédard, Kröger, \& Destexhe, 2006; Freeman, 2004; Pritchard, 1992), making high-frequency $\gamma_{\mathrm{H}}$ signals difficult to record at the surface of the scalp (but see Ball et al., 2008; Lenz et al., 2008). Furthermore, $\gamma_{H}$ activity recorded at the scalp is susceptible to noise from scalp (Fu et al., 2006; Goncharova et al., 2003), facial (Whitham et al., 2008), and eye movement (Yuval-Greenberg, Tomer, Keren, Nelken, \& Deouell, 2008) muscles. These noise sources, coupled with the well-known localization issues due to the inverse problem, limit neurocognitive scalp EEG research. These issues have been verified in many experimental and computational models of the interaction between the skull and the EEG (Cooper, Winter, Crow, \& Walter, 1965; Geisler \& Gerstein, 1961; Abraham \& Marsan, 1958; Williams \& Parsons-Smith, 1950) and have shaped the way human EEG research has been performed for the past several decades.

Although MEG, implanted electrodes, and intraoperative intracranial electrophysiology overcome some of these limitations, they are sensitive to other confounding issues. Scalp MEG requires subjects to sit with their heads motionless in rooms shielded from electromagnetic noise, and the MEG is less sensitive to radial dipole sources in the crowns of gyri (Cohen \& Cuffin, 1991). Intraoperative electrophysiology during neurosurgical procedures is not only invasive but also limited by cognitive and EEG changes associated with abnormal neural tissue, pharmacological manipulations during anesthesia, and small number of patients available for study. Here we report the viability of the hemicraniectomy model. The results of our analysis provide the first systematic quantification of the effects of the skull on behavioral EEG that could help bridge findings from human intracranial and extracranial electrophysiology.

Absent intervening skull, our electrodes were physically closer to the surface of the brain and free from the smoothing effects of bone and nonneural tissue, resulting in larger EEG signal and improved spatial independence. Without a measure of electrode conductivity, we cannot rule out the possibility of a systematic bias in conductivity between hemicraniectomy and skull electrodes. Such a bias would result in apparent signal differences. However, there are several aspects of our data that suggest that conductivity differences are not a significant variable. We recorded all electrodes from the same amplifier with comparable skin resistance. The signal-to-noise ratio indicates good signal quality over the hemicraniectomy, and we can assume that the effects of noise on interelectrode correlations should be minimized. The hemicraniectomy and the skull power spectra have the same basic shape, which suggest that their signals have the same general levels of noise. Finally, because the hemicraniectomy electrodes are closer to the cortex (the signal source), one might predict on a simple amplitude basis that-barring any filtering or smoothing by the skull-neighboring hemicraniectomy electrodes would be more similar. However, we found evidence that the hemicraniectomy electrodes are more independent supporting the notion we were recording from more restricted neural populations.

We found larger task-relevant ERPs and ERSPs with more robust high-frequency activity over the site of hemicraniectomy. Furthermore, movement-related $\beta$ and $\gamma_{\mathrm{H}}$ activity are visible at the single-trial level over the hemicraniectomy site. Previous research has demonstrated reliable BMI control in humans using scalp EEG $\beta$ (Wolpaw $\&$ McFarland, 2004) and intracranial $\gamma_{\mathrm{H}}$ (Ball et al. 2008; Miller et al., 2007) frequencies. We believe this is the first instance showing that coupling between two different frequencies in a single electrode can also be used as an index for movement. Specifically, this GBI is better correlated with movement than the $\beta$ or $\gamma_{H}$ bands alone. Of note, the scalp $\gamma_{\mathrm{H}}$ band is compromised by signal strength decrease and muscle noise from a host of sources (eye, facial, scalp, and pharyngeal muscles), so this boost in movement prediction using the GBI is not as evident at the level of the scalp. This GBI adds to the growing literature (Canolty et al., 2006) that information obtained through interfrequency coupling provides a valuable metric to assess neurobehavioral network dynamics that can complement time-series analysis.

The enhanced window to EEG activity provided by a hemicraniectomy may provide an important model to test the accuracy and efficacy of electrophysiological sourcelocalization models and component extraction methods such as principal- and independent-components analysis. Several studies have used EEG and MEG data collected from subjects with smaller skull defects (e.g., burr holes, craniectomy scars) to test a variety of inverse modeling methods (Yoshinaga, Kobayashi, Hoshida, Kinugasa, \& Ohtuska, 2008; Li, Wang, Zhu, \& He, 2007; Bénar \& Gotman, 2002; Oostenveld \& Oostendorp, 2002). Subjects with a hemicraniectomy provide a larger window to the cortexand thus a greater number of electrodes with less spatial smoothing - to use for testing these models. Recent research has also focused on examining individual skull thickness variability on a variety of ERP and time-frequency components (Hagemann, Hewig, Walter, \& Naumann, 2008; Frodl et al., 2001; Pfefferbaum, 1990). Hemicraniectomy subjects may provide a new way of examining such relationships.

A recent study reported movement-related $\gamma_{\mathrm{H}}$ activity over scalp EEG (Darvas et al., in press). $\gamma_{\mathrm{H}}$ was extracted from the average activity of voxels over premotor and motor cortex using an inverse solution of EEG electrode activity mapped onto the cortical surface. This method allows $\gamma_{\mathrm{H}}$ activity extraction from a local region of cortex in a manner that may not be possible to do in a single scalp 
EEG electrode due to smoothing effects, whereas the craniectomy inherently provides a direct window to the cortical surface with decreased smoothing. This is relevant clinically, as the development of long-term, accurate BMI for paralyzed patients is of utmost importance. The hemicraniectomy recording method may prove to be an important bridge between the spatial and the spectrotemporal quality of ECoG and the long-term stability of scalp EEG in BMI research. Given the increasing use of hemicraniectomy for neurosurgical purposes and the ability to test these subjects repeatedly as outpatients, we suggest that this model may have applications to a wide variety of cognitive research questions.

\section{Acknowledgments}

This research was supported by the American Psychological Association Diversity Program in Neuroscience (5-T32-MH18882 to B.V.), the UC Systemwide Biotechnology Research and Education Program (UCBREP to L. V.), the Fondation pour la Recherche Medicale to A. B. C., and the National Institute of Neurological Disorders and Stroke (NS21135-22S1 to B. V., NS050173 to G. T. M., and NS21135 to R. T. K.). the authors thank Maya Cano, Adeen Flinker, and Noa Fogelson for assistance with data collection; Ryan Canolty and Adeen Flinker for critical discussions; and Wes Clapp, Christina Karns, and Jessica Voytek for feedback on the manuscript.

Reprint requests should be sent to Bradley Voytek, Helen Wills Neuroscience, University of California, Berkeley, 132 Barker Hall, Berkeley, CA 94720-3190, or via e-mail: bradley.voytek@ gmail.com.

\section{REFERENCES}

Abraham, K., \& Marsan, C. (1958). Patterns of cortical discharges and their relation to routine scalp electroencephalography. Electroencephalography and Clinical Neurophysiology, 10, 447-461.

Ball, T., Demandt, E., Mutschler, I., Neitzel, E., Mehring, C., Vogt, K., et al. (2008). Movement related activity in the high gamma range of the human EEG. Neuroimage, 41, 302-310.

Ball, T., Kern, M., Mutschler, I., Aertsen, A., \& Schulze-Bonhage, A. (2009). Signal quality of simultaneously recorded invasive and non-invasive EEG. Neuroimage, 46, 708-716.

Bédard, C., Kröger, H., \& Destexhe, A. (2006). Does the 1/f frequency scaling of brain signals reflect self-organized critical states? Physical Review Letters, 97, 118102.

Bénar, C. G., \& Gotman, J. (2002). Modeling of post-surgical brain and skull defects in the EEG inverse problem with the boundary element method. Clinical Neurophysiology, 113, 48-56.

Berger, H. (1929). Über das Elektrenkephalogramm des Menchen. Archives für Psychiatrie, 87, 527-570.

Bruns, A. (2004). Fourier-, Hilbert- and wavelet-based signal analysis: Are they really different approaches? Journal of Neuroscience Methods, 137, 321-332.

Canolty, R. T., Edwards, E., Dalal, S. S., Soltani, M., Nagarajan, S. S., Kirsch, H. E., et al. (2006). High gamma power is phase-locked to theta oscillations in human neocortex. Science, 313, 1626-1628.

Cobb, W., \& Sears, T. (1960). A study of the transmission of potentials after hemispherectomy. Electroencephalography and Clinical Neurophysiology, 12, 371-833.
Cobb, W. A., Guiloff, R. J., \& Cast, J. (1979). Breach rhythm: The EEG related to skull defects. Electroencephalography and Clinical Neurophysiology, 47, 251-271.

Cohen, D., \& Cuffin, B. N. (1991). EEG versus MEG localization accuracy: Theory and experiment. Brain Topography, 4, 95-103.

Cooper, R., Winter, A., Crow, H., \& Walter, W. (1965). Comparison of subcortical, cortical and scalp activity using chronically indwelling electrodes in man. Electroencephalography and Clinical Neurophysiology, 18, 217-228.

Crone, N. E., Miglioretti, D. L., Gordon, B., \& Lesser, R. P. (1998). Functional mapping of human sensorimotor cortex with electrocorticographic spectral analysis. II. Event-related synchronization in the gamma band. Brain, 121, 2301-2315.

Crone, N. E., Miglioretti, D. L., Gordon, B., Sieracki, J. M., Wilson, M. T., Uematsu, S., et al. (1998). Functional mapping of human sensorimotor cortex with electrocorticographic spectral analysis. I. Alpha and beta event-related desynchronization. Brain, 121, 2271-2299.

Dalal, S. S., Guggisberg, A., Edwards, E., Sekihara, K., Findlay, A., Canolty, R. T., et al. (2008). Five-dimensional neuroimaging: Localization of the time-frequency dynamics of cortical activity. Neuroimage, 40, 1686-1700.

Darvas, F., Scherer, R., Ojemann, J. G., Rao, R. P., Miller, K. J., \& Sorensen, L. B. (in press). High gamma mapping using EEG. Neuroimage.

de Lange, F. P., Jensen, O., Bauer, M., \& Toni, I. (2008). Interactions between posterior gamma and frontal alpha/ beta oscillations during imagined actions. Frontiers in Human Neuroscience, 2, 7.

Delorme, A., \& Makeig, S. (2004). EEGLAB: An open source toolbox for analysis of single-trial EEG dynamics including independent component analysis. Journal of Neuroscience Methods, 134, 9-21.

DeLucchi, M., Garoutee, B., \& Aird, R. (1962). The scalp as an electroencephalographic averager. Electroencephalography and Clinical Neurophysiology, 14, 191-196.

Edwards, E., Soltani, M., Deouell, L. Y., Berger, M., \& Knight, R. T. (2005). High gamma activity in response to deviant auditory stimuli recorded directly from human cortex. Journal of Neurophysiology, 94, 4269-4280.

Freeman, W. (2004). Origin, structure, and role of background EEG activity: Part I. Analytic amplitude. Clinical Neurophysiology, 115, 2077-2088.

Frodl, T., Meisenzahl, E. M., Müller, D., Leinsinger, G., Juckel, G., Hahn, K., et al. (2001). The effect of the skull on event-related P300. Clinical Neurophysiology, 112, 1773-1776.

Fu, M. J., Daly, J. J., \& Çavuşoğlu, M. (2006). A detection scheme for frontalis and temporalis muscle EMG contamination of EEG data. Conference Proceedings: Annual International Conference of the IEEE Engineering in Medicine and Biology Society, 1, 4514-4518.

Geisler, C. D., \& Gerstein, G. L. (1961). The surface EEG in relation to its sources. Electroencephalography and Clinical Neurophysiology, 13, 927-934.

Goncharova, I. I., McFarland, D. J., Vaughn, T. M., \& Wolpaw, J. R. (2003). EMG contamination of EEG: Spectral and topographical characteristics. Clinical Neurophysiology, 114, 1580-1593.

Hagemann, D., Hewig, J., Walter, C., \& Naumann, E. (2008). Skull thickness and magnitude of EEG alpha activity. Clinical Neurophysiology, 119, 1271-1280.

Hochberg, L., Serruya, M., Friehs, G., Mukand, J., Saleh, M., Caplan, A., et al. (2006). Neuronal ensemble control of 
prosthetic devices by a human with tetraplegia. Nature, 442, 164-171.

Hofmeijer, J., Amelink, G., Algra, A., Van Gijn, J., Macleod, M., Kappelle, L., et al. (2006). Hemicraniectomy after middle cerebral artery infarction with life-threatening Edema trial (HAMLET). Protocol for a randomised controlled trial of decompressive surgery in space-occupying hemispheric infarction. Trials, 7, 29.

Huang, A., Tu, Y., Tsai, Y., Chen, Y., Hong, W., Yang, C., et al. (2008). Decompressive craniectomy as the primary surgical intervention for hemorrhagic contusion. Journal of Neurotrauma, 25, 1347-1354.

Juttler, E., Schwab, S., Schmiedek, P., Unterberg, A., Hennerici, M., Woitzik, J., et al. (2007). Decompressive surgery for the treatment of malignant infarction of the middle cerebral artery (DESTINY): A randomized, controlled trial. Stroke, 38, 2518-2525.

Koessler, L., Maillard, L., Benhadid, A., Vignal, J., Felblinger, J., Vespignani, H., et al. (2009). Automated cortical projection of EEG sensors: Anatomical correlation via the international 10-10 system. Neuroimage, 46, 69-72.

Lenz, D., Jeschke, M., Schadow, J., Naue, N., Ohl, F., \& Herrmann, C. (2008). Human EEG very high frequency oscillations reflect the number of matches with a template in auditory short-term memory. Brain Research, 1220, 81-92.

Li, J., Wang, K., Zhu, S., \& He, B. (2007). Effects of holes on EEG forward solutions using a realistic geometry head model. Journal of Neural Engineering, 4, 197-204.

Luck, S. J. (2005). An introduction to the event-related potential technique. Cambridge, MA: MIT Press.

Metting van Rijn, A. C., Peper, A., \& Grimbergen, C. A. (1990). High-quality recording of bioelectric events: Part I. Interference reduction, theory and practice. Medical and Biological Engineering and Computing, 28, 389-397.

Miller, K., Dennijs, M., Shenoy, P., Miller, J., Rao, R., \& Ojemann, J. (2007). Real-time functional brain mapping using electrocorticography. Neuroimage, 37, 504-507.

Millett, D. (2001). Hans Berger: From psychic energy to the EEG. Perspectives in Biology and Medicine, 44, 522-542.

Neuper, C., Wörtz, M., \& Pfurtscheller, G. (2006). ERD/ERS patterns reflecting sensorimotor activation and deactivation. Progress in Brain Research, 159, 211-222.

Nunez, P. L., \& Srinivasan, R. (2005). Electric fields of the brain: The neurophysics of EEG (2nd ed.). New York: Oxford University Press.
Okamoto, M., \& Dan, I. (2005). Automated cortical projection of head-surface locations for transcranial functional brain mapping. Neuroimage, 26, 18-28.

Oostenveld, R., \& Oostendorp, T. (2002). Validating the boundary element method for forward and inverse EEG computations in the presence of a hole in the skull. Human Brain Mapping, 17, 179-192.

Pfefferbaum, A. (1990). Model estimates of CSF and skull influences on scalp-recorded ERPs. Alcohol, 7, 479-482.

Pfurtscheller, G., \& Lopes da Silva, F. H. (1999). Event-related EEG/MEG synchronization and desynchronization: Basic principles. Clinical Neurophysiology, 110, 1842-1857.

Pritchard, W. S. (1992). The brain in fractal time: 1/f-like power spectrum scaling of the human electroencephalogram. International Journal of Neuroscience, 66, 119-129.

Solnik, S., DeVita, P., Rider, P., Long, B., \& Hortobágyi, T. (2008). Teager-Kaiser Operator improves the accuracy of EMG onset detection independent of signal-to-noise ratio. Acta of Bioengineering and Biomechanics, 10, 65-68.

Vahedi, K., Vicaut, E., Mateo, J., Kurtz, A., Orabi, M., Guichard, J., et al. (2007). Sequential-design, multicenter, randomized, controlled trial of early decompressive craniectomy in malignant middle cerebral artery infarction (DECIMAL Trial). Stroke, 38, 2506-2517.

Whitham, E., Lewis, T., Pope, K., Fitzgibbon, S., Clark, C., Loveless, S., et al. (2008). Thinking activates EMG in scalp electrical recordings. Clinical Neurophysiology, 119, 1166-1175.

Williams, D., \& Parsons-Smith, G. (1950). Cortical rhythms not seen in the electroencephalogram. Brain, 73, 191-202.

Wolpaw, J., \& McFarland, D. J. (2004). Control of a two-dimensional movement signal by a noninvasive brain-computer interface in humans. Proceedings of the National Academy of Sciences, U.S.A., 101, 17849-17854.

Yang, X., Wen, L., Li, G., Zhan, R., Ma, L., \& Liu, W. (2009). Contralateral subdural effusion secondary to decompressive craniectomy performed in patients with severe traumatic brain injury: Incidence, clinical presentations, treatment and outcome. Medical Principles and Practice, 18, 16-20.

Yoshinaga, H., Kobayashi, K., Hoshida, T., Kinugasa, K., \& Ohtuska, Y. (2008). Magnetoencephalogram in a postoperative case with a large skull defect. Pediatric Neurology, 39, 48-51.

Yuval-Greenberg, S., Tomer, O., Keren, A. S., Nelken, I., \& Deouell, L. Y. (2008). Transient induced gamma-band response in EEG as a manifestation of miniature saccades. Neuron, 58, 429-441. 